https://helda.helsinki.fi

\title{
NMR tensors in planar hydrocarbons of increasing size
}

\section{Ikäläinen, Suvi}

2009

Ikäläinen , S , Lantto , P , Manninen , P \& Vaara , J 2009 , ' NMR tensors in planar

hydrocarbons of increasing size ' , Physical Chemistry Chemical Physics , vol. 11 , no. 48 , pp. 11404-11414 . https://doi.org/10.1039/b919860a

http://hdl.handle.net/10138/27906

https://doi.org/10.1039/b919860a

publishedVersion

Downloaded from Helda, University of Helsinki institutional repository.

This is an electronic reprint of the original article.

This reprint may differ from the original in pagination and typographic detail.

Please cite the original version. 


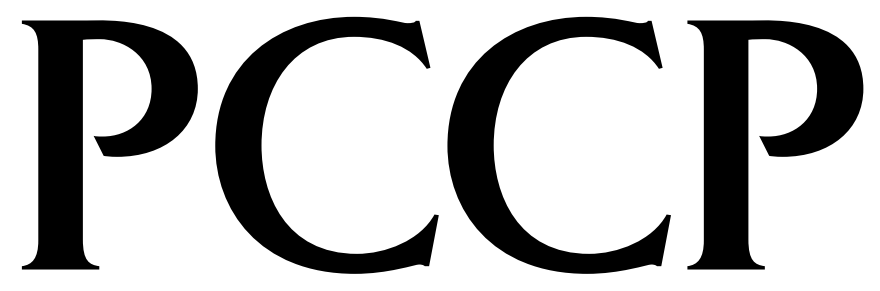

\section{Physical Chemistry Chemical Physics}

\section{COVER ARTICLE}

Vaara et al.

NMR tensors in planar hydrocarbons of increasing size
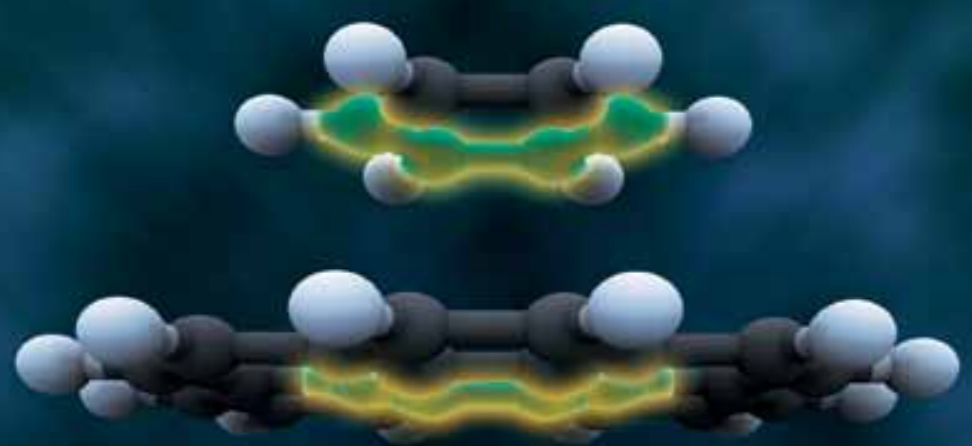

\section{COMMUNICATION}

Mons et al.

Gas phase folding of an (Ala) ${ }_{4}$ neutral peptide chain: spectroscopic evidence for the

formation of a $\beta$-hairpin $\mathrm{H}$-bonding pattern 


\title{
NMR tensors in planar hydrocarbons of increasing size $\dagger$
}

\author{
Suvi Ikäläinen, ${ }^{a}$ Perttu Lantto, ${ }^{b}$ Pekka Manninen ${ }^{c}$ and Juha Vaara ${ }^{* a d}$ \\ Received 23rd September 2009, Accepted 21st October 2009 \\ First published as an Advance Article on the web 7th November 2009 \\ DOI: $10.1039 / \mathrm{b} 919860 a$
}

\begin{abstract}
${ }^{13} \mathrm{C}$ nuclear shielding and ${ }^{13} \mathrm{C}-{ }^{13} \mathrm{C}$ spin-spin coupling tensors were calculated using density functional theory linear response methods for a series of planar hydrocarbons. As calculation of the spin-spin coupling is computationally demanding for large molecules due to demands placed on basis-set quality, novel, compact completeness-optimized (co) basis sets of high quality were employed. To maximize the predictive value of the data, the convergence of the co basis sets was compared to well-known basis-set families. The selection of the exchange-correlation functional was performed based on the available experimental data and coupled-cluster calculations for ethene and benzene. The series of hydrocarbons, benzene, coronene, circumcoronene and circumcircumcoronene, was chosen to simulate increasingly large fragments of carbon nanosheets. It was found that the nuclear shielding and the one-, two-, and three-bond spin-spin coupling constants, as well as the corresponding anisotropies with respect to the direction normal to the plane, approach convergence as the number of carbon atoms in the fragment is increased. Predictions of the investigated properties can then be done for the limit of large planar hydrocarbons or carbon nanosheets. From the results obtained with a judicious choice of the functional, PBE, and co basis close to convergence, limiting values are estimated as follows: $\sigma=54 \pm 1 \mathrm{ppm}$ [corresponding to the chemical shift of $134 \mathrm{ppm}$ with methane $\left(\mathrm{CH}_{4}\right)$ as a reference], $\Delta \sigma=207 \pm 4 \mathrm{ppm},{ }^{1} J=59.0 \pm 0.5 \mathrm{~Hz}, \Delta^{1} J=-1.5 \pm 0.5 \mathrm{~Hz},{ }^{2} J=0.2 \pm 0.4 \mathrm{~Hz}$ $\Delta^{2} J=-4.6 \pm 0.2 \mathrm{~Hz},{ }^{3} J=6 \pm 1 \mathrm{~Hz}$, and $\Delta^{3} J=3 \pm 1 \mathrm{~Hz}$.
\end{abstract}

\section{Introduction}

Nuclear magnetic resonance (NMR) $)^{1,2}$ is an important mode of spectroscopy, which can be used to determine molecular structure. The nuclear shielding tensor $\boldsymbol{\sigma}$ and the indirect spin-spin coupling tensor $\mathbf{J}$ are parameters that determine NMR spectra. Nuclear shielding refers to the altered magnetic field experienced by a nucleus, resulting from the motion of the electrons in the external field of the NMR spectrometer. Spin-spin coupling, on the other hand, corresponds to the change in the magnetic field experienced by a nucleus that is affected by neighboring magnetic nuclei.

Recently developed techniques have made it possible to produce two-dimensional sheets of graphene, ${ }^{3}$ which raises interest in the magnetic and NMR properties of such carbon nanostructures. ${ }^{4-6}$ Investigation of the electronic structure of graphene using solid-state methods with periodic boundary conditions has revealed a gapless semiconductor band structure. ${ }^{7}$ Finite graphene fragments, single-layer nanosheets,

${ }^{a}$ Laboratory of Physical Chemistry, Department of Chemistry, P.O. Box 55 (A.I. Virtasen aukio 1), FIN-00014 University of Helsinki, Finland

${ }^{b}$ NMR Research Group, Department of Physics, P.O. Box 3000, FIN-90014 University of Oulu, Finland

${ }^{c}$ CSC-IT Center for Science Ltd., P.O. Box 405, FIN-02101 Espoo, Finland

${ }^{d}$ NMR Research Group, Department of Physics, P.O. Box 3000, FIN-90014 University of Oulu, Finland. E-mail: juha.vaara@oulu.fi $\dagger$ Electronic supplementary information (ESI) available: Tabulated exponents of the co-b and co-r basis sets, results obtained with the locally dense basis-set approximation, and illustration of the calculated HOMO-LUMO gap. See DOI: 10.1039/b919860a are systems with a finite band gap that can be related to large planar hydrocarbons. The NMR of pure and doped graphene has been investigated theoretically, based on the behavior of Dirac fermions with linear electronic dispersion relation. ${ }^{8}$ In contrast, we are not aware of any prior first-principles NMR studies of either the infinite sheet or large planar fragments thereof. Also, experimental NMR studies have not yet been conducted on such systems. For NMR of three-dimensional graphite, see, e.g., refs. 9 and 10 .

Periodic first-principles methods for magnetic properties have been reported for $\boldsymbol{\sigma}$ (refs. 11 and 12) and $\mathbf{J}$ (ref. 13). At the moment, the quantum chemical approach, ${ }^{14,15}$ applicable to finite molecules, still offers some benefits as compared to the periodic models. These include the localized atomic allelectron basis sets that are well-suited to hyperfine interactions, and the availability of the technology for carrying out correlated $a b$ initio and hybrid density functional theory (DFT) calculations. With rapidly increasing computational resources it is tempting to pursue the magnetic properties of extended structures by increasingly large cluster calculations. Here we do so, targeting the NMR properties of large planar hydrocarbons.

Starting from $\mathrm{C}_{6} \mathrm{H}_{6}$, one may create a series of increasingly large superbenzenes by adding consecutive planar layers of benzene rings around the carbon six-ring. The shielding and spin-spin coupling tensors depend on the hyperfine operators that are localized at the atomic nuclei, as well as the global electronic structure of the molecule. By applying DFT for the $\boldsymbol{\sigma}$ and $\mathbf{J}$ tensors for benzene, coronene $\left(\mathrm{C}_{24} \mathrm{H}_{12}\right)$, circumcoronene $\left(\mathrm{C}_{54} \mathrm{H}_{18}\right)$ and circumcircumcoronene $\left(\mathrm{C}_{96} \mathrm{H}_{24}\right)$ 
depicted in Fig. 1, we investigate whether the local and global features balance so that trends can be observed that allow prediction of these properties for the large-system limit. A calibration study using ethene $\left(\mathrm{C}_{2} \mathrm{H}_{4}\right)$ and benzene is first conducted to select the quantum-chemical models. Evaluation of the $\boldsymbol{\sigma}$ and $\mathbf{J}$ tensors for ethene and benzene, both theoretically and experimentally, has been performed previously in various studies, e.g., refs. 16 and 17. Among the three larger systems, we are only aware of one computational NMR study, reporting Hartree-Fock level ${ }^{13} \mathrm{C}$ chemical shifts of coronene. $^{18}$ Ref. 19 reports a related theoretical evaluation of the $\mathbf{J}$ tensors of the $\mathrm{C}_{60}$ molecule. Nuclear shieldings of increasingly large fragments of single-wall carbon nanotubes (SWNTs) have been evaluated computationally using DFT methods in ref. 20. It was found that $\mathrm{C}_{30}$-capped SWNT fragments in particular represent acceptable models of infinite systems, as the investigated properties converge quite quickly with the length of the fragment.

Calculation of the $\mathbf{J}$ tensor is exceptionally difficult due to the stringent demands placed on the basis sets and the level of theory that are required to obtain qualitatively correct results. At the nonrelativistic level, spin-spin coupling involves four
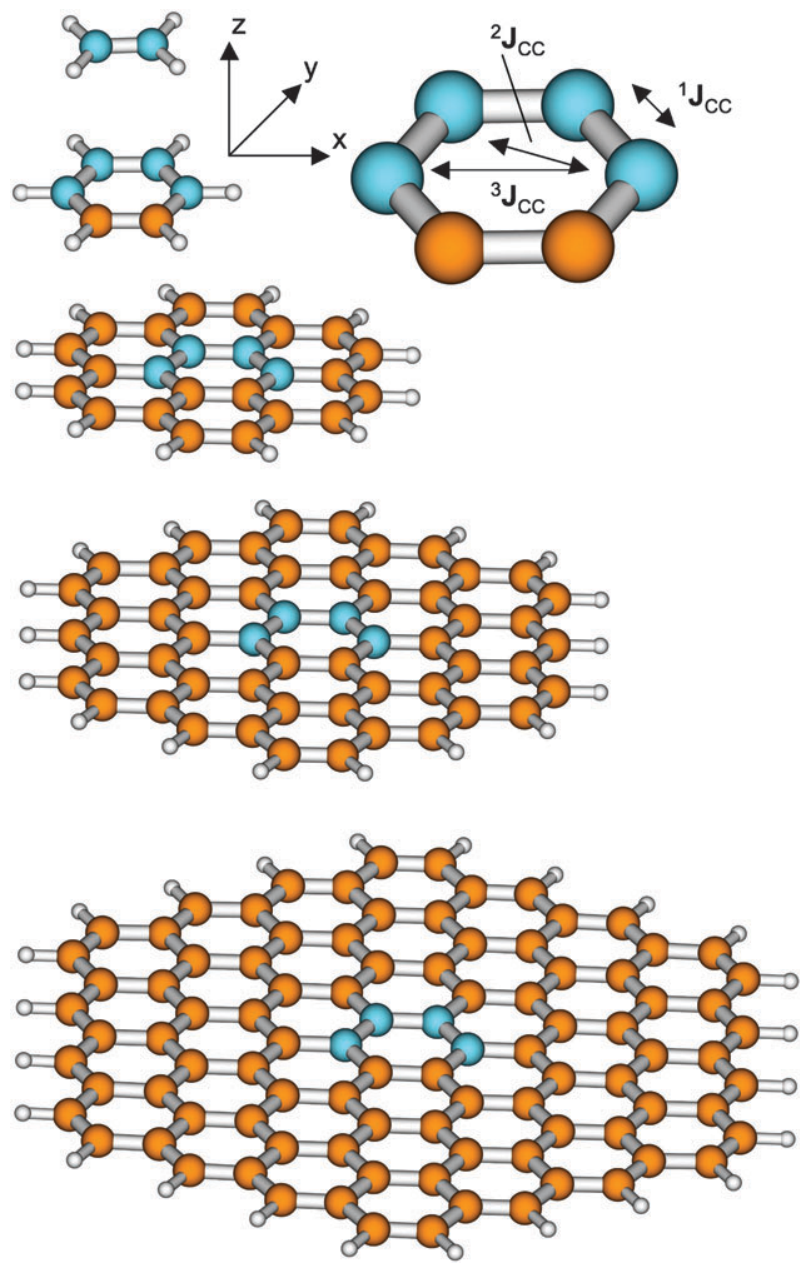

Fig. 1 Studied molecules: ethene, benzene, coronene, circumcoronene, and circumcircumcoronene. The differently colored carbons indicate the atoms between which the spin-spin coupling tensor was calculated. The shielding tensor was calculated for the innermost carbon atoms. different physical mechanisms, which require accurate description of the electron density near the nuclei. Some of these mechanisms involve triplet perturbation operators, which renders restricted Hartree-Fock methods useless. ${ }^{15}$ Due to the size of the larger molecules studied presently, conventional basis sets giving acceptable results cannot be used. The novel method of completeness optimization (co, ref. 21) was thus adopted to generate computationally efficient basis sets that give results close to the basis set limit. These sets were compared to the pcJ- $n$ basis sets, ${ }^{22}$ which have been designed specifically for DFT calculations of spin-spin coupling. In order to maintain predictive quality for the large-system limit, significant attention has been paid to both the basis-set convergence and selection of the exchange-correlation functional.

\section{Theory}

\subsection{Spin-spin coupling and nuclear shielding}

The NMR properties depend on hyperfine interactions that are localized at the nuclei. On the other hand, there is a dependence on the global electronic structure of the molecule through the relevant excitation energies and excited-state wave functions. These features can be summarized in the representation of the second-order, linear-response parts $T$ of $\boldsymbol{\sigma}$ and $\mathbf{J}$ in a Rayleigh-Schrödinger-type perturbation theory expression

$$
T=\sum_{n} \frac{\left\langle 0\left|\hat{O}_{1}\right| n\right\rangle\left\langle n\left|\hat{O}_{2}\right| 0\right\rangle}{E_{0}-E_{n}}+\text { c.c. },
$$

where $|0\rangle$ and $|n\rangle$ refer to the labels of the ground and excited states of the unperturbed system, respectively, with $E_{0}$ and $E_{n}$ the corresponding energies. The $\hat{O}_{i}$ denote the hyperfine and Zeeman operators relevant for the property at hand. They couple the ground state to excited states of different spatial and spin (singlet or triplet) symmetries.

Spin-spin coupling can be characterized by four hyperfine operators; diamagnetic and paramagnetic nuclear spin-electron orbit interactions (DSO and PSO), the Fermi contact operator (FC), and the spin-dipole operator (SD). For closed-shell molecules, after taking into account the selection rules for transitions involving the electron spin, the four operators give rise to five different terms in the $\mathbf{J}$ tensor. ${ }^{23}$ The total tensor can be written as

$$
\mathbf{J}_{\mathrm{KL}}=\mathbf{J}_{\mathrm{KL}}^{\mathrm{DSO}}+\mathbf{J}_{\mathrm{KL}}^{\mathrm{PSO}}+\mathbf{J}_{\mathrm{KL}}^{\mathrm{SD}}+\mathbf{J}_{\mathrm{KL}}^{\mathrm{FC}}+\mathbf{J}_{\mathrm{KL}}^{\mathrm{SD} / \mathrm{FC}}
$$

for a pair of magnetic nuclei KL. $\mathbf{J}^{\mathrm{DSO}}$ is calculated as a ground-state expectation value, while the remaining terms are calculated as linear response functions involving first-order perturbed wave functions. $\mathbf{J}^{\mathrm{DSO}}$ and $\mathbf{J}^{\mathrm{PSO}}$ involve singlet perturbation operators, whereas $\mathbf{J}^{\mathrm{SD}}, \mathbf{J}^{\mathrm{FC}}$, and the cross-term $\mathbf{J}^{\mathrm{SD} / \mathrm{FC}}$ include triplet perturbations.

The four first terms in eqn (2) contribute to the isotropic rotational average, which for the spin-spin coupling constant is $J=\frac{1}{3} \operatorname{Tr} \mathbf{J}$. In the case of planar systems we expect distinct NMR properties for directions in-plane and perpendicular to the carbon sheet. Hence, the tensorial properties of NMR interactions are of interest and we also calculate the anisotropy of the coupling, $\Delta J=J_{z z}-J_{\perp}$. Here, $J_{z z}$ and $J_{\perp}=\frac{1}{2}\left(J_{x x}+J_{y y}\right)$ 
are the components of the coupling tensor perpendicular to and within the plane of the molecule, respectively. The DSO, $\mathrm{PSO}, \mathrm{SD}$ and $\mathrm{SD} / \mathrm{FC}$ terms contribute to the anisotropic properties of $\mathbf{J}$, while the FC term is fully isotropic.

The nuclear shielding is calculated as a response to the Zeeman interaction with the external magnetic field. ${ }^{24}$ In this context, first- and second-order, dia- and paramagnetic terms arise, respectively. Gauge invariance of the results is imposed by using gauge-including atomic orbitals (GIAOs). ${ }^{25,26}$ The paramagnetic shielding only involves a linear response with respect to singlet perturbation operators, the orbital Zeeman and PSO interactions, and is typically computationally less demanding than spin-spin coupling. Analogous to $\mathbf{J}$, both the shielding constant $\sigma$ and the anisotropy $\Delta \sigma$ are reported.

\subsection{Basis sets}

It has been found in previous investigations ${ }^{14,22,27-31}$ that the addition of high-exponent $s$ functions to a basis set can greatly improve the basis-set convergence of $\mathbf{J}$. The tight $s$ functions are needed to saturate the FC term, which in one- and two-bond coupling constants is usually the predominating contribution. It was emphasized in ref. 22 by Jensen, however, that the PSO and SD contributions, which often dominate three-bond coupling constants, require the addition of tight $p$ functions to promote convergence. The SD term additionally places a demand for tight $d$ and $f$ functions. Using these criteria, a new category of basis sets, the pcJ- $n$ sets, was formulated. ${ }^{22}$ These basis sets are constructed by addition of tight $s, p, d$, and $f$ functions to polarization-consistent (pc- $n$ ) basis sets, which have been optimized for use with DFT methods. $^{32}$ A sequence of five pcJ- $n$ basis sets is available ( $n=0-5$ ), which should be capable of converging to within $0.1 \%$ of the basis-set limit. ${ }^{22}$

Completeness optimization was introduced by Manninen and Vaara in ref. 21 as a novel method for generating Gaussian basis sets based only on their mathematical properties. An important tool used in completeness optimization is the concept of the completeness profile, defined as ${ }^{33}$

$$
Y(\zeta)=\sum_{m}\left\langle g(\zeta) \mid \chi_{m}\right\rangle^{2}
$$

where $\{\chi\}$ is a set of orthonormalized basis functions for a given angular momentum $l$ and $g(\zeta)$ is a "test" Gaussian type orbital (GTO) with the exponent $\zeta . g(\zeta)$ is used to analyze the completeness of $\{\chi\}$, and the value of $Y(\zeta)$ can be made to equal 1 for all $\zeta$ in a Gaussian basis set with an infinite number of functions. A measure for the deviation from completeness is ${ }^{21}$

$$
\tau=\int_{\zeta_{\min }}^{\zeta_{\max }}[1-Y(\zeta)] \mathrm{d} \zeta
$$

$Y(\zeta)$ can be visualized on a $[\log (\zeta), Y(\zeta)]$ plot, in which case the profile of a basis set that is complete for a certain range of $\zeta$ will equal 1 within this range and 0 elsewhere, creating a plateau-type figure. Completeness profiles of the completenessoptimized basis sets used can be seen in Fig. 2.

In completeness optimization, energetic criteria are abandoned in basis set generation. ${ }^{21}$ Instead, primitive sets of GTOs are generated with the purpose of spanning, with a suitable compromise of accuracy (small $\tau$ ) and economy (small number of functions), a pre-selected exponent range $\left[\zeta_{\min }, \zeta_{\max }\right]$ in which $Y(\zeta) \approx 1$. With this technique, significant reduction in the size of basis sets with no loss in the accuracy of the results has been demonstrated for magnetic properties. ${ }^{21,34}$ This is in practice accomplished with the Kruununhaka software, ${ }^{35}$ where one specifies the exponent region in which the basis set should be complete, i.e., the overlap with arbitrary GTOs in the same exponent range approaches unity. ${ }^{21}$ Subsequently, the number of functions is reduced while maintaining the range. This will increase $\tau$ and cause ripples in the plateau region of the completeness profile. The adequate exponent range and smoothness of the plateau can be determined experimentally by carrying out trial calculations of the investigated property in a small test molecule. Hence, the quality of the basis set is determined by the exponent interval $\left[\zeta_{\min }, \zeta_{\max }\right]$ and allowed $\tau$ for each angular momentum subspace of the different elements. In a recent paper, ${ }^{34}$ we employed co basis sets in the calculation of laser-induced NMR splittings in large hydrocarbons similar to the present systems.

\section{Calculations}

Calculations of $\boldsymbol{\sigma}$ and $\mathbf{J}$ were conducted with the DALTON ${ }^{36}$ and ACES-II ${ }^{37}$ programs at DFT and $a b$ initio levels, respectively. The studied molecules, ethene, benzene, coronene, circumcoronene, and circumcircumcoronene are depicted in Fig. 1. Ethene was used as a small test molecule to investigate the performance of the different basis sets and functionals. First, a series of calculations of $\boldsymbol{\sigma}$ and $\mathbf{J}$ using the $\mathrm{BLYP}^{38,39}$ functional and co basis sets, pcJ- $n$ sets, and selected conventional basis sets was performed.

The uncontracted co basis sets used in this work were generated using a scheme similar to that in ref. 34. First, a reference basis set was created for carbon that spans the same exponent range as the aug-cc-pCV $5 \mathrm{Z}$ basis, ${ }^{40}$ a set which gives results for $\mathbf{J}_{\mathrm{CC}}\left(\mathrm{C}_{2} \mathrm{H}_{4}\right)$ close to convergence, allowing only a small deviation from completeness. The exponent range was then expanded separately for each $l$ value until convergence was reached. A range giving results for $\mathbf{J}$ and $\Delta J$ within $0.1 \mathrm{~Hz}$ from the limiting value was selected for the basis set co-b. The basis set co-r was then obtained by reducing the number of basis functions in the co-b set, keeping the results within $0.2 \mathrm{~Hz}$ from the co-b results. For hydrogen, the co-b has similarly a large exponent range and, in this case, the second step involved not only a reduction of the number of functions, but of the exponent range as well. Table 1 shows the structures and exponent ranges of the basis sets co-b and co-r. The exponent values are listed in the Supporting Information.

Because of limitations in the DALTON program, calculations for the largest system, circumcircumcoronene, are not feasible with the co-r basis set. As the shielding and coupling involve highly local hyperfine operators, the locally dense basis-set concept ${ }^{41,42}$ may be employed. The co-r* basis was constructed by using co-r only for the innermost benzene ring of the molecule, while the conventional, contracted def2-SVP set $^{43}$ was used for the remaining atoms. In co-r**, the region 

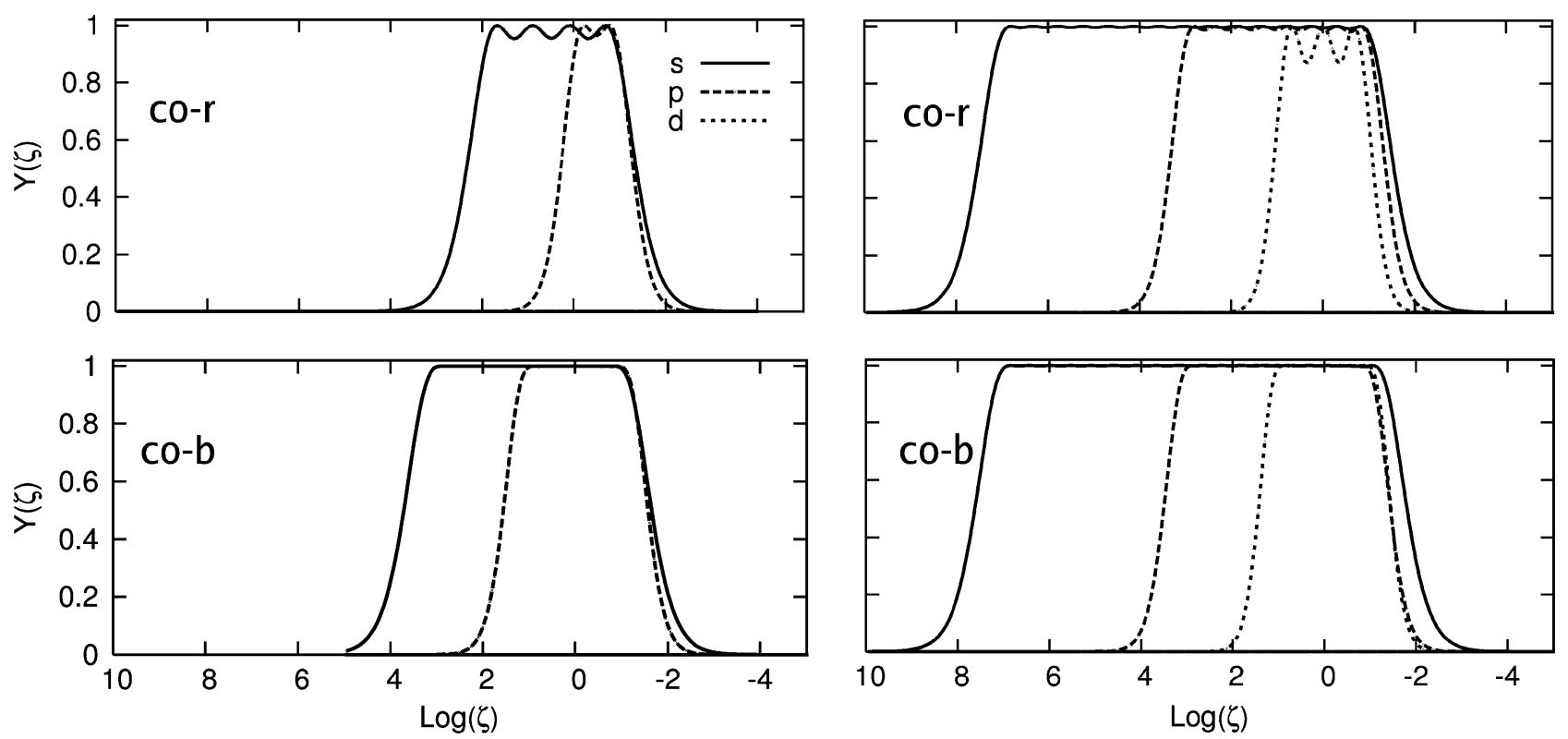

Fig. 2 Completeness profiles of the completeness-optimized basis sets, with hydrogen basis sets on the left and carbon sets on the right.

Table 1 Structure and exponent ranges for basis sets co-r and co-b. The number of functions, $n$, is also given for the different systems

\begin{tabular}{lll}
\hline Property & co-r & co-b \\
\hline $\mathrm{C}:$ functions & $15 s 8 p 3 d$ & $18 s 11 p 7 d$ \\
$\mathrm{C}:\left[\zeta_{\min }-\zeta_{\max }\right]$ & $s: 0.1-10000000$ & $s: 0.1-10000000$ \\
& $p: 0.1-1000$ & $p: 0.1-1000$ \\
& $d: 0.1-10$ & $d: 0.1-10$ \\
$\mathrm{H}:$ functions & $4 s 2 p$ & $11 s 7 p$ \\
$\mathrm{H}:\left[\zeta_{\text {min }}-\zeta_{\text {max }}\right]$ & $s: 0.1-100$ & $s: 0.1-1000$ \\
$n\left(\mathrm{C}_{2} \mathrm{H}_{4}\right)$ & $p: 0.1-1$ & $p: 0.1-10$ \\
$n\left(\mathrm{C}_{6} \mathrm{H}_{6}\right)$ & 148 & 300 \\
$n\left(\mathrm{C}_{24} \mathrm{H}_{12}\right)$ & 384 & 708 \\
$n\left(\mathrm{C}_{54} \mathrm{H}_{18}\right)$ & 1416 & 2448 \\
$n\left(\mathrm{C}_{96} \mathrm{H}_{24}\right)$ & 3096 & - \\
${ }^{a}$ co-r ${ }^{* *}$ for circumcircumcoronene. See text for details. \\
\hline
\end{tabular}

treated with the larger basis set was extended to two innermost layers of carbon atoms.

The exploratory calculations for ethene were used to select basis sets that are sufficiently small for treating the larger systems, but still give results that are close to the basis-set limit. After the basis-set calibration, calculations using the coupled-cluster singles and doubles (CCSD) method and the DFT functionals BLYP, B3LYP, ${ }^{39,44,45}$ BHandHLYP, ${ }^{39,46}$ $\mathrm{PBE}$, and $\mathrm{PBE} 0,{ }^{47}$ were carried out for both ethene and benzene for comparison of the different functionals. It is well-known that DFT produces relatively good results for $\mathbf{J}_{\mathrm{CC}}{ }^{48-50}$ The $\boldsymbol{\sigma}$ and $\mathbf{J}$ tensors were then calculated with PBE as well as the pcJ-1 and co basis sets for the larger systems. For systems larger than ethene, also the two- and three-bond couplings, ${ }^{2} \mathbf{J}$ and ${ }^{3} \mathbf{J}$, were calculated in addition to the one-bond coupling ${ }^{1} \mathbf{J}$.

For ethene and benzene we used the best available theoretical geometries based on high-level ab initio calculations, the $r_{z}$ geometry for ethene ${ }^{51}$ and $r_{e}$ for benzene..$^{52}$ The geometries of coronene, circumcoronene, and circumcircumcoronene were optimized using the Turbomole software. ${ }^{43}$ The BP8 $6^{38,53}$ functional was used with the def2-TZVP ${ }^{54}$ basis in these calculations. Nuclear shielding was also calculated for methane with the co-r basis and the PBE functional to provide a chemical shift reference. The geometry for $\mathrm{CH}_{4}$ was also optimized at the BP86/def2-TZVP level. A result of $\sigma=$ $188.33 \mathrm{ppm}$ was obtained.

\section{Results and discussion}

\subsection{Basis-set convergence}

The present work is the first application of the co basis sets to large-scale calculations of the conventional NMR parameters, $\boldsymbol{\sigma}$ and $\mathbf{J}$. A comparison of the convergence properties with the conventional, energy-optimized basis sets is therefore in place. The results for $\sigma, \Delta \sigma, J$, and $\Delta J$ for ethene using BLYP and different basis set families are presented in Table 2. Various correlation-consistent basis sets, cc-pVXZ, ${ }^{55}$ cc-pCVXZ, ${ }^{40}$ aug-cc-pVXZ, ${ }^{56}$ aug-cc-pCVXZ, ${ }^{40}$ Jensen's pcJ- $n$ sets, and co-sets were used. $\sigma$ and $J$ are also illustrated in Fig. 3 and 4 , respectively.

Convergence of the nuclear shielding is generally easier to achieve than for spin-spin coupling. $\sigma$ and $\Delta \sigma$ converge smoothly and in most cases monotonically to common limiting values around 42 and $174 \mathrm{ppm}$, respectively, within the different basis set families. The correlation-consistent basis sets without tight core-valence functions (cc-pVXZ and aug-cc-pVXZ) exhibit slower convergence than the other basis sets. At the other extreme, the convergence of the pcJ- $n$ series is very rapid. Both completeness-optimized sets are practically at the basis set limit, with co-r giving the best performance compared to other sets of the same size.

Spin-spin coupling parameters, $J$ and $\Delta J$, also converge towards common limits, 69 and $20 \mathrm{~Hz}$, respectively, for all basis set families. There is more initial fluctuation in the results 


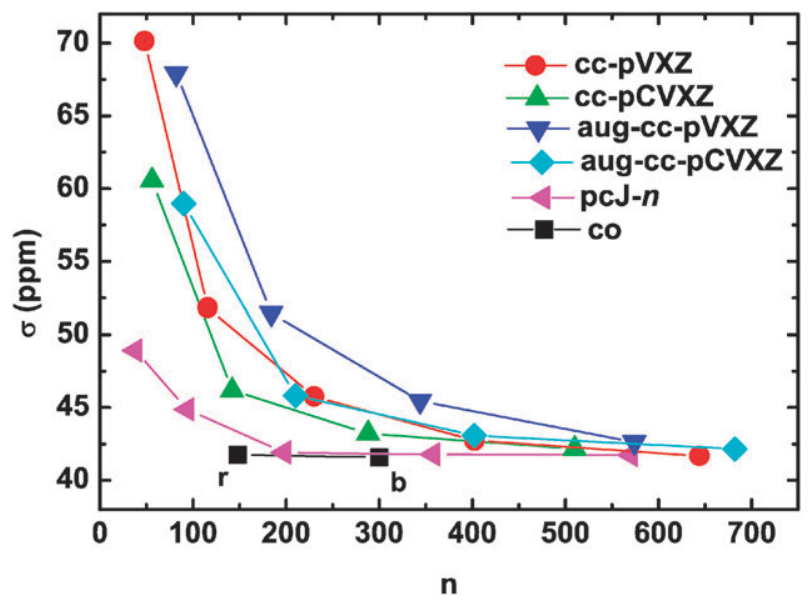

Fig. $3{ }^{13} \mathrm{C}$ nuclear shielding constant in ethene as a function of the number of basis functions with various basis-set families using the BLYP method.

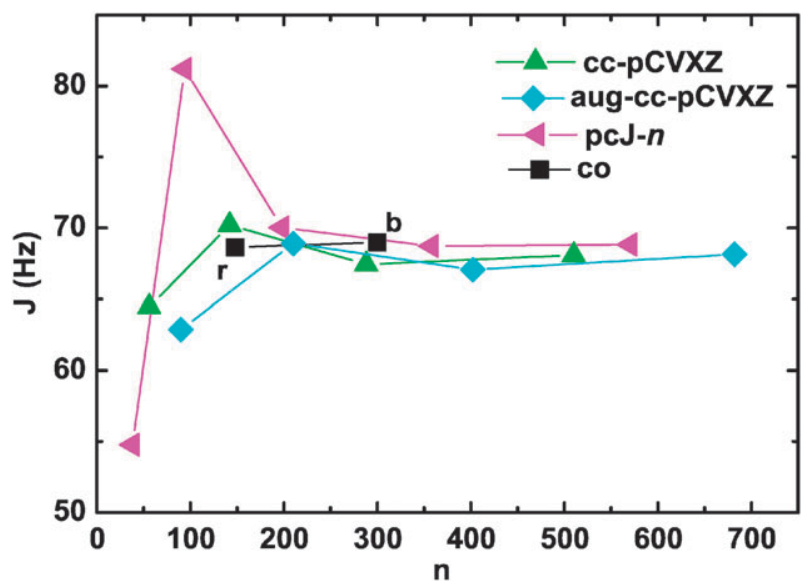

Fig. $4{ }^{13} \mathrm{C}-{ }^{13} \mathrm{C}$ spin-spin coupling constant in ethene as a function of the number of basis functions with various basis-set families using the BLYP method.

for $J$ and its anisotropy than for nuclear shielding. The cc-pVXZ and aug-cc-pVXZ basis sets have been omitted in Fig. 4, because of the generally poor performance in calculating spin-spin couplings due to their incompleteness in the region of tight functions.

Concerning the choice of the parameters in the co basis sets, there are greater demands placed on the extent of the exponent range in the tight $s$ region by the FC interaction as compared to, e.g., the properties investigated in ref. 34. It was found that not only the top of the plateau region in the completeness profile, but also the initial increase at the root of the $s$-type profile, is relevant for $\mathbf{J}^{\mathrm{FC}}$. Fig. 4 shows that the completenessoptimized basis sets give reliable values for $J$ with relatively small basis sets. Both co basis sets give results quite close to each other. The co-r set displays the best performance at around 150 functions for ethene.

Table 3 shows the different contributions to $\mathbf{J}$ with the basis sets pcJ-2, pcJ-4, co-b, and co-r, using the PBE functional. All basis sets give rather similar results, with the FC term being the predominant one for ${ }^{1} J$, and both PSO and SD/FC being
Table 2 Computed nuclear shielding constants and ${ }^{13} \mathrm{C}-{ }^{13} \mathrm{C}$ spin-spin coupling, $\sigma$ and $J$, respectively, as well as the corresponding anisotropies $(\Delta \sigma$ and $\Delta J)$ for ethene using the BLYP functional and various basis sets. $n$ denotes the number of basis functions. Shieldings in ppm, couplings in $\mathrm{Hz}$

\begin{tabular}{llllll}
\hline Basis set & $n$ & $\sigma$ & $\Delta \sigma^{a}$ & $J$ & $\Delta J^{a}$ \\
\hline cc-pVDZ & 48 & 70.16 & 154.85 & 84.09 & 22.87 \\
cc-pVTZ & 116 & 51.83 & 166.43 & 77.01 & 7.12 \\
cc-pVQZ & 230 & 45.77 & 171.23 & 63.35 & 12.20 \\
cc-pV5Z & 402 & 42.74 & 173.67 & 72.05 & 17.64 \\
cc-pV6Z & 644 & 41.68 & 174.60 & 69.06 & 19.51 \\
cc-pCVDZ & 56 & 60.55 & 161.61 & 64.46 & 13.72 \\
cc-pCVTZ & 142 & 46.16 & 171.03 & 70.20 & 16.29 \\
cc-pCVQZ & 288 & 43.21 & 173.21 & 67.45 & 20.03 \\
cc-pCV5Z & 510 & 42.16 & 174.13 & 68.09 & 20.08 \\
aug-cc-pVDZ & 82 & 67.91 & 156.88 & 135.04 & 20.26 \\
aug-cc-pVTZ & 184 & 51.46 & 167.73 & 55.35 & 7.09 \\
aug-cc-pVQZ & 344 & 45.45 & 171.51 & 64.06 & 14.63 \\
aug-cc-pV5Z & 574 & 42.60 & 173.80 & 72.65 & 17.57 \\
aug-cc-pCVDZ & 90 & 58.96 & 163.55 & 62.88 & 12.87 \\
aug-cc-pCVTZ & 210 & 45.81 & 171.84 & 68.90 & 14.63 \\
aug-cc-pCVQZ & 402 & 43.08 & 173.42 & 67.06 & 19.81 \\
aug-cc-pCV5Z & 682 & 42.14 & 174.16 & 68.15 & 20.06 \\
pcJ-0 & 38 & 48.91 & 172.22 & 54.77 & 19.93 \\
pcJ-1 & 94 & 44.86 & 171.15 & 81.20 & 20.30 \\
pcJ-2 & 198 & 41.91 & 174.34 & 70.03 & 17.53 \\
pcJ-3 & 358 & 41.77 & 174.44 & 68.72 & 19.71 \\
pcJ-4 & 570 & 41.73 & 174.48 & 68.81 & 19.88 \\
co-r & 148 & 41.76 & 176.17 & 68.64 & 20.38 \\
co-b & 300 & 41.58 & 174.40 & 68.99 & 20.26
\end{tabular}

a Anisotropies defined as $\Delta T=T_{z z}-\frac{1}{2}\left(T_{x x}+T_{y y}\right)$, where the $z$ direction is normal to the plane of the molecule and $x$ and $y$ are within the plane.

important for $\Delta^{1} J$. The total spin-spin coupling and anisotropy are slightly smaller with co-r than with the larger co-b and pcJ-4 basis sets, which is mostly due to a more negative ${ }^{1} J^{\mathrm{PSO}}$ and a smaller $\mathrm{SD} / \mathrm{FC}$ contribution in $\Delta^{1} J$. pcJ-2 gives total values for ${ }^{1} J$ and $\Delta^{1} J$ that are larger than with pcJ-4 or co-b, because of small overestimation of the FC term in the total coupling as well as the $\mathrm{SD}$ and $\mathrm{SD} / \mathrm{FC}$ terms in the anisotropy. For comparison, we also include the CCSD/co-b results. It is found that the $\mathrm{PBE} / \mathrm{co}-\mathrm{b}$ level of theory reproduces the $a b$ initio data for the total ${ }^{1} J$ and $\Delta^{1} J$ relatively well. A closer inspection reveals that there are differences of up to $1.5 \mathrm{~Hz}\left({ }^{1} J\right)$ and $2.7 \mathrm{~Hz}\left(\Delta^{1} J\right)$ between the PBE and CCSD levels in the individual PSO, SD, and FC contributions, but they largely cancel out for the total observables. Due to its small size, ethene is more sensitive to changes in basis-set quality than the larger systems that will be examined. In the larger molecules, basis-set superposition makes convergence even better than for ethene.

\subsection{DFT functionals}

Results for particularly the spin-spin coupling as obtained using different DFT functionals fall into a relatively large range. ${ }^{14,30,31,48,49}$ Consequently, to obtain meaningful predictions for the larger systems it makes sense to carry out a critical investigation of different functionals for small, related molecules, using the available experimental data and $a b$ initio results as reference material. Results for spin-spin coupling and nuclear shielding, along with anisotropies, for ethene and benzene with the basis set co-b and the different DFT 
Table $3 J$ and $\Delta J$ for the ${ }^{13} \mathrm{C}-{ }^{13} \mathrm{C}$ spin-spin coupling tensor in ethene broken down into the different contributions (in $\mathrm{Hz}$ ) with different functionals, CCSD, and different basis sets

\begin{tabular}{|c|c|c|c|c|c|c|c|c|c|}
\hline Method & Basis set & $n$ & Property & Total & DSO & PSO & SD & $\mathrm{FC}$ & $\mathrm{SD} / \mathrm{FC}$ \\
\hline \multirow[t]{7}{*}{ PBE } & pcJ-2 & 198 & $\begin{array}{l}{ }^{1} J \\
\Delta^{1} J\end{array}$ & $\begin{array}{l}72.93 \\
30.20\end{array}$ & $\begin{array}{l}0.07 \\
-2.46\end{array}$ & $\begin{array}{l}-9.95 \\
14.78\end{array}$ & $\begin{array}{l}4.77 \\
7.57\end{array}$ & 78.04 & $\overline{10.31}$ \\
\hline & \multirow[t]{2}{*}{ pcJ-4 } & \multirow[t]{2}{*}{570} & ${ }^{1} J$ & 71.80 & 0.07 & $\begin{array}{l}14.10 \\
-9.92\end{array}$ & 4.66 & $\overline{76.99}$ & 10.51 \\
\hline & & & $\Delta^{1} J$ & 29.70 & -2.46 & 14.76 & 7.41 & - & 9.99 \\
\hline & \multirow[t]{2}{*}{ co-r } & \multirow[t]{2}{*}{148} & ${ }^{1} J$ & 71.24 & 0.07 & -10.13 & 4.59 & 76.71 & - \\
\hline & & & $\Delta^{1} J$ & 29.33 & -2.46 & 15.01 & 7.30 & - & 9.48 \\
\hline & \multirow[t]{2}{*}{ co-b } & \multirow[t]{2}{*}{300} & ${ }^{1} J$ & 71.86 & 0.07 & -9.97 & 4.58 & 77.18 & - \\
\hline & & & $\Delta^{1} J$ & 29.70 & -2.46 & 14.78 & 7.30 & - & 10.08 \\
\hline \multirow[t]{2}{*}{ BLYP } & \multirow[t]{2}{*}{$\mathrm{co-b}$} & \multirow[t]{2}{*}{300} & ${ }^{1} J$ & 69.06 & 0.07 & -10.12 & 6.70 & 87.35 & - \\
\hline & & & $\Delta^{1} J$ & 17.80 & -2.46 & 15.14 & 5.62 & - & -0.49 \\
\hline \multirow[t]{2}{*}{ B3LYP } & \multirow[t]{2}{*}{ co-b } & \multirow[t]{2}{*}{300} & ${ }^{1} J$ & 73.40 & 0.07 & -10.36 & 4.28 & 79.42 & - \\
\hline & & & $\Delta^{1} J$ & 25.22 & -2.46 & 15.45 & 6.73 & - & 5.50 \\
\hline \multirow[t]{2}{*}{ BHandHLYP } & \multirow[t]{2}{*}{$\mathrm{co}-\mathrm{b}$} & \multirow[t]{2}{*}{300} & ${ }^{1} J$ & 88.26 & 0.06 & -10.48 & 6.41 & 92.27 & - \\
\hline & & & $\Delta^{1} J$ & 46.70 & -2.46 & 15.76 & 9.87 & - & 23.53 \\
\hline \multirow[t]{2}{*}{ PBE0 } & \multirow[t]{2}{*}{ co-b } & \multirow[t]{2}{*}{300} & ${ }^{1} J$ & 84.00 & 0.07 & -10.12 & 6.70 & 87.35 & - \\
\hline & & & $\Delta^{1} J$ & 49.76 & -2.46 & 15.12 & 10.44 & — & 26.67 \\
\hline \multirow[t]{2}{*}{ CCSD } & \multirow[t]{2}{*}{ co-b } & \multirow[t]{2}{*}{300} & ${ }^{1} J$ & 70.49 & 0.07 & -9.17 & $3.01^{a}$ & 76.58 & - \\
\hline & & & $\Delta^{1} J$ & $-^{b}$ & -2.46 & 14.06 & $4.60^{a}$ & - & $-^{b}$ \\
\hline
\end{tabular}

functionals, as well as the ab initio CCSD method are found in Table 4, where experimental values are also given. Results for $\sigma$ show the systematic deshielding characteristic to DFT, as compared to either experimental or ab initio data. Chemical shifts with respect to a suitable reference compound would be reproduced much better. ${ }^{59,60}$ BLYP and PBE give the best results for ${ }^{1} J$, as judged by the agreement with experiment, while the hybrid functionals B3LYP and BHandHLYP, on the one hand, and PBE0 on the other hand, lead to overestimation that is increasing systematically with the percentage of including the exact Hartree-Fock exchange $(20 \%, 50 \%$, and $25 \%$ for B3LYP, BHandHLYP, and PBE0, respectively). Similar systematic trends are noted in all the presently calculated parameters. BLYP, B3LYP, and PBE perform better than BHandHLYP and PBE0 for ${ }^{2} J$ and ${ }^{3} J$ in benzene.

Table 3 presents the different contributions to ${ }^{1} J$ and $\Delta^{1} J$ for ethene using the different DFT functionals and the CCSD method with the basis set co-b. From the table, PBE is closest to CCSD results for the total value of ${ }^{1} J$ and all contributions that may be compared, except $\Delta^{1} J^{\mathrm{SD}}$, for which BLYP performs best. DSO contributions are equivalent with all methods, and PSO results are all quite close to each other. The effect of systematic overestimation with the percentage of including exact exchange is seen in the FC term. The differences in the anisotropy are dominated by the SD/FC term, which changes by as much as $27.76 \mathrm{~Hz}$ between the DFT methods.

The anisotropies of $J$ are extremely difficult to measure experimentally, ${ }^{30}$ which makes comparison of functionals based on the experimental anisotropy data problematic. Comparison to the liquid-crystal NMR results ${ }^{16,17}$ implies that the hybrid functionals would have some advantage over GGAs for the coupling anisotropies but no definite conclusions may be drawn for the present systems. It was found in ref. 50 that PBE, which has a more rigorous physical background ${ }^{47}$ than the other functionals used here, gives results close to CCSD quality when calculating spin-spin couplings.
It is well-known that the basis-set convergence is slower for correlated $a b$ initio methods than for single-determinantal models, such as DFT. Consequently, the present CCSD/co-b data are further away from their basis-set limit than the DFT/co-b data from their corresponding limit. The difference arises particularly from the larger importance of high-angular momentum polarization-type functions for the $a b$ initio methods than for DFT. Experience shows, however, that this tendency is much less severe for the NMR properties than for energetics. Consequently, the present preferences as regards DFT functionals, partially on the basis of comparison with CCSD using the relatively large co-b basis, should be justified. Based on this, as well as the previous success of PBE in calculations of spin-spin couplings, this functional was chosen for the larger systems in this study.

\subsection{Larger molecules}

4.3.1 Basis sets. Locally dense basis sets were tested for coronene, circumcoronene, and circumcircumcoronene, with the intention of validating their use for the largest of these molecules. The Electronic Supplementary Information (ESI $\dagger$ ) contains tabulated shieldings and couplings for these systems as obtained using the PBE functional with the co-r* and co-r** basis sets, as well as co-r for comparison. Fig. 5 illustrates the data for $\sigma$ and ${ }^{1} J$. The locally dense basis sets reproduce the results of the full co-r basis accurately for the molecules other than coronene, for which relatively large differences occur between co-r* and co-r. For circumcoronene and circumcircumcoronene, the co-r* and co-r** values are quite close to each other. Both results are close to the best co-r data for the former system. This indicates that it is within the locally dense basis set concept sufficient to employ the full basis set for the neighboring carbon layer in addition to the innermost six-ring.

The results for $\boldsymbol{\sigma}$ and $\mathbf{J}$ for the series of planar molecules from benzene to circumcircumcoronene using PBE and pcJ-1, co-b, and co-r basis sets are given in Table 5. pcJ-1 was chosen 
Table 4 Nuclear shielding and spin-spin coupling constants and anisotropies for ethene and benzene with the basis set co-b and different DFT functionals

\begin{tabular}{|c|c|c|c|c|c|}
\hline \multirow[b]{2}{*}{ System } & \multirow[b]{2}{*}{ Functional } & \multicolumn{2}{|c|}{ Shielding } & \multicolumn{2}{|c|}{$\underline{\text { Spin-spin coupling }}$} \\
\hline & & $\sigma$ & $\Delta \sigma$ & ${ }^{1} J$ & $\Delta^{1} J$ \\
\hline \multirow[t]{9}{*}{$\mathrm{C}_{2} \mathrm{H}_{4}$} & BLYP & 41.58 & 174.40 & 69.06 & 17.80 \\
\hline & B3LYP & 43.77 & 178.16 & 73.40 & 25.22 \\
\hline & BHandHLYP & 47.79 & 180.56 & 88.26 & 46.70 \\
\hline & PBE & 44.76 & 176.75 & 71.86 & 29.70 \\
\hline & PBE0 & 47.72 & 180.14 & 83.61 & 51.41 \\
\hline & CCSD & 68.30 & 165.72 & $70.49^{a}$ & $-b$ \\
\hline & Exp. $^{c}$ & 68.27 & 160.15 & 67.50 & 27.74 \\
\hline & & \multicolumn{2}{|c|}{ Shielding } & \multicolumn{2}{|c|}{ One-bond spin-spin coupling } \\
\hline & & $\sigma$ & $\Delta \sigma$ & ${ }^{1} J$ & $\Delta^{1} J$ \\
\hline \multirow{14}{*}{$\mathrm{C}_{6} \mathrm{H}_{6}$} & BLYP & 40.00 & 187.43 & 56.17 & 0.79 \\
\hline & B3LYP & 42.08 & 192.92 & 59.83 & 4.59 \\
\hline & BHandHLYP & 45.65 & 197.72 & 71.66 & 16.62 \\
\hline & PBE & 44.39 & 188.79 & 57.75 & 5.64 \\
\hline & PBE0 & 47.01 & 194.58 & 66.43 & 15.14 \\
\hline & Exp. & $57.11^{d}$ & $180^{e}$ & $55.87^{f}$ & $17.50^{f}$ \\
\hline & & \multicolumn{2}{|c|}{ Two-bond spin-spin coupling } & \multicolumn{2}{|c|}{ Three-bond spin-spin coupling } \\
\hline & & ${ }^{2} J$ & $\Delta^{2} J$ & ${ }^{3} J$ & $\Delta^{3} J$ \\
\hline & BLYP & -0.34 & -5.94 & 10.66 & 5.14 \\
\hline & B3LYP & -1.92 & -8.77 & 11.35 & 8.08 \\
\hline & BHandHLYP & -7.30 & -19.41 & 15.66 & 19.21 \\
\hline & PBE & -3.23 & -9.23 & 12.41 & 8.53 \\
\hline & PBE0 & -7.75 & -17.53 & 15.80 & 17.08 \\
\hline & Exp..$^{f}$ & -2.47 & -3.90 & 10.11 & 9.50 \\
\hline
\end{tabular}

${ }^{a}$ SD contribution calculated with the co-r basis set. ${ }^{b}$ The ACES-II program does not report the SD/FC cross-term contribution necessary for $\Delta J .{ }^{c}$ Ref. 16, liquid crystal solution. ${ }^{d}$ Ref. 57 , gas-phase. ${ }^{e}$ Ref. 58 , solid-state. ${ }^{f}$ Ref. 17 , liquid crystal solution.

as a compact reference basis because it belongs to a basis-set family that is establishing itself in the DFT calculation of NMR parameters. pcJ-2 would have been clearly closer to the basis-set limit, but at a computational cost that would have been prohibitive for the largest system. The pcJ-1 results are distinctly further removed from the co-b data than the co-r results. For ${ }^{1} J$, pcJ-1 gives larger values than the co sets, and the difference grows with the size of the molecule. It is evident from Table 5 that, similar to ethene, co-r and co-b give very similar results for benzene and coronene, and the differences become smaller in the larger of the two systems. It may then be assumed that the results obtained for the two larger systems are reliable, and close to the basis-set limit.

Orendt et al. reported a solid-state experiment on coronene, ${ }^{18}$ with the room-temperature values of the chemical shift with respect to tetramethylsilane (TMS) as well as shielding anisotropy equal to $120 \mathrm{ppm}$ and $216 \mathrm{ppm}$, respectively. Converting our PBE/co-r shielding constant, 49.25 ppm into the chemical shift with respect to, first, $\mathrm{CH}_{4}$ results in $139.08 \mathrm{ppm}$. As the absolute ${ }^{13} \mathrm{C}$ shielding constant of TMS is 7 ppm smaller than that of methane in low-pressure gas, ${ }^{61}$ the present datum corresponds roughly to the shift of $132 \mathrm{ppm}$ with respect to TMS. Even considering that thermal as well as medium effects are present in the experimental data but not in our model, the calculated shift is clearly larger than the experimental result. On the basis of the data in Table 4 for $\mathrm{C}_{2} \mathrm{H}_{4}$ and $\mathrm{C}_{6} \mathrm{H}_{6}$, the difference is too large to result from choosing a particular DFT functional. In contrast, the calculated anisotropy (211 ppm) is in satisfactory agreement with the experiment. For comparison, the experimental chemical shift of $\mathrm{C}_{60}$ is reported at $143 \mathrm{ppm}$ with respect to
TMS. ${ }^{62,63}$ The shielding anisotropy in $\mathrm{C}_{60}$ with respect to the most shielded direction has been reported as $\Delta \sigma=163 \mathrm{ppm}$ in ref. 64. Both parameters deviate clearly from the values obtained presently for the planar hydrocarbons. The theoretical one-bond spin-spin coupling within/between the pentagons of $\mathrm{C}_{60}$ are $62 / 77 \mathrm{~Hz}$ as obtained with the B3LYP functional and entry-level basis set, ${ }^{19}$ i.e., larger than for the current systems.

4.3.2 Large-system limit. Fig. 6 shows the one-, two-, and three-bond spin-spin coupling as well as nuclear shielding constants, and the corresponding anisotropies for benzene, coronene, circumcoronene, and circumcircumcoronene using PBE and the co-r basis set. All the parameters except for ${ }^{1} J$ and $\sigma$ display, in the three largest molecules, an oscillatory convergence with the system size around the respective limiting values. ${ }^{1} J$ continues to grow for these systems, but signs of convergence to a limiting value are already starting to appear and all values fall to a fairly narrow range around $58 \mathrm{~Hz}$. $\sigma$ grows in the three smaller systems, but levels off after circumcoronene. From Fig. 6, it appears that for larger planar systems, these properties should have similar values. Calculations of still larger members of the series would be of interest for the definite verification of the convergence. Unfortunately, such computations are beyond the resources currently accessible for us, even with the co basis sets. While the converged values are subject to the present choice of DFT functional and - to a much lesser extent - the quality of the co-r basis set, the overall convergence behavior is expected to be invariant to these parameters. Our methodology does not allow definite conclusions to be made concerning the relevance 

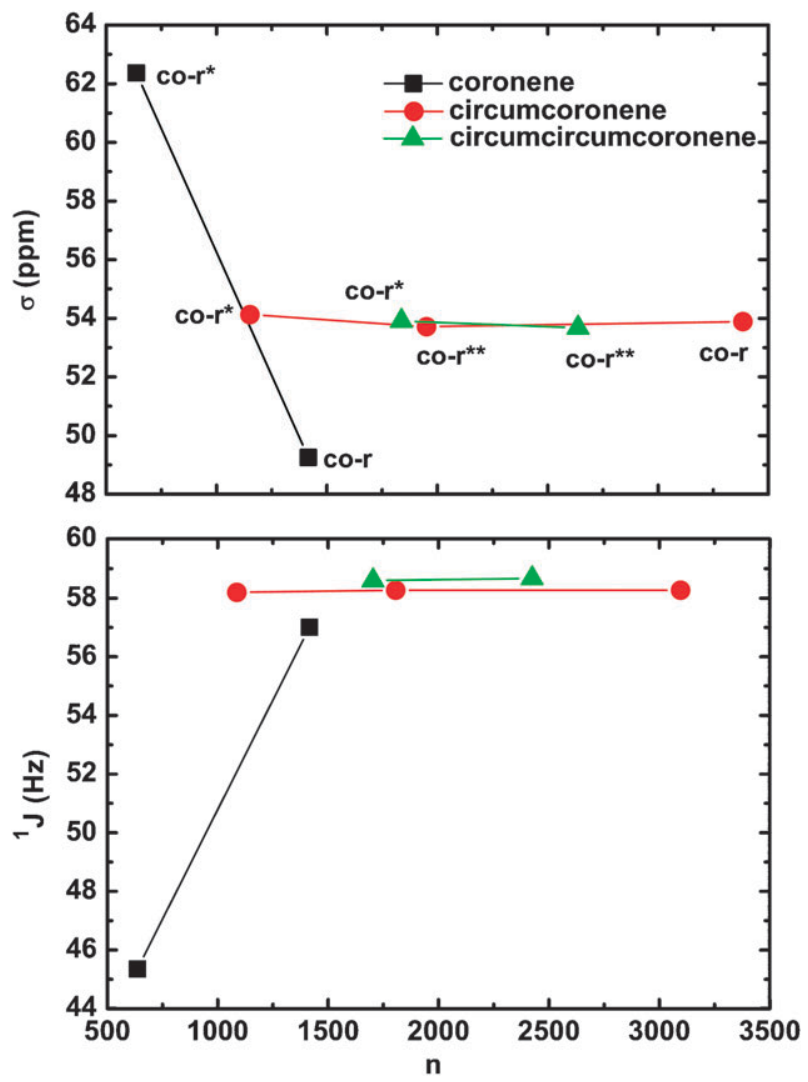

Fig. 5 Performance of locally dense basis sets (co-r* and co-r**) as compared to the full basis set (co-r) for the ${ }^{13} \mathrm{C}$ nuclear shielding constant and ${ }^{13} \mathrm{C}-{ }^{13} \mathrm{C}$ one-bond spin-spin coupling constant, as a function of the number of basis functions. The PBE functional was used for coronene, circumcoronene, and circumcircumcoronene. See text for details.

of the limit of large but finite system properties to the infinite graphene sheet. The present method only accounts for the orbital contribution to nuclear shielding, omitting any Knight shift effects ${ }^{2}$ due to metallic conduction electrons. For undoped graphene at room temperature, the Knight shift contribution has been phenomenologically estimated at below 0.4 ppm. ${ }^{8}$ Nevertheless, our data should at least constitute a plausible starting point of the analysis of eventual experiments for graphene.

The limiting behavior apparent from our results may be compared with the heuristically estimated values for the isotropic chemical shift referenced to TMS and $\Delta \sigma$ of graphene in ref. 4, $128 \mathrm{ppm}$ and $163 \mathrm{ppm}$, respectively. Our corresponding first-principles data are $134 \mathrm{ppm}$ (with respect to $\mathrm{CH}_{4}$ ) and $207 \mathrm{ppm}$. While the anisotropy values disagree, there is a remarkable agreement between the isotropic chemical shifts, taken the difference of the absolute shielding constants of the TMS and $\mathrm{CH}_{4}$ reference systems (vide supra).

The contributions to $\mathbf{J}$ from the different physical mechanisms using the co-r basis set and PBE functional are listed in Table 6, along with the HOMO-LUMO gaps for the systems. Benzene differs from all the larger molecules. For ${ }^{1} J$, the other terms converge, while the FC term appears to continue growing, and is thus responsible for the remaining trend in this parameter in our largest molecules. All other parameters
Table $5{ }^{13} \mathrm{C}$ shielding constants $\sigma$, shielding anisotropies $\Delta \sigma,{ }^{13} \mathrm{C}-{ }^{13} \mathrm{C}$ spin-spin coupling constants ${ }^{1,2,3} J$ and the corresponding anisotropies $\Delta^{1,2,3} J$ for benzene, coronene, circumcoronene, and circumcircumcoronene using the PBE functional and different basis sets. Shieldings in ppm, couplings in $\mathrm{Hz}$. Couplings within the innermost carbon hexagon are considered. Anisotropies are with respect to the direction perpendicular to the plane of the molecule

\begin{tabular}{|c|c|c|c|c|c|c|c|c|}
\hline \multirow[b]{2}{*}{ Basis set } & \multicolumn{2}{|l|}{$\mathrm{C}_{6} \mathrm{H}_{6}$} & \multicolumn{2}{|c|}{$\mathrm{C}_{24} \mathrm{H}_{12}$} & \multicolumn{2}{|c|}{$\mathrm{C}_{54} \mathrm{H}_{18}$} & \multicolumn{2}{|c|}{$\mathrm{C}_{96} \mathrm{H}_{24}{ }^{a}$} \\
\hline & $\sigma^{b}$ & $\Delta \sigma$ & $\sigma^{b}$ & $\Delta \sigma$ & $\sigma^{b}$ & $\Delta \sigma$ & $\sigma^{b}$ & $\Delta \sigma$ \\
\hline pcJ-1 & 47.81 & 185.73 & 46.48 & 205.16 & 57.92 & 200.84 & - & - \\
\hline co-r & 45.10 & 190.23 & 49.25 & 211.28 & 53.89 & 205.94 & 53.67 & 208.95 \\
\hline \multirow[t]{2}{*}{ co-b } & 44.39 & 188.79 & 49.06 & 211.40 & - & - & - & - \\
\hline & ${ }^{1} J$ & $\Delta^{1} J$ & ${ }^{1} J$ & $\Delta^{1} J$ & ${ }^{1} J$ & $\Delta^{1} J$ & ${ }^{1} J$ & $\Delta^{1} J$ \\
\hline pcJ-1 & 59.95 & 1.93 & 64.72 & -1.15 & 65.93 & -0.42 & - & - \\
\hline co-r & 57.47 & 5.63 & 57.01 & -1.64 & 58.27 & -1.00 & 58.67 & -1.32 \\
\hline \multirow[t]{2}{*}{ co-b } & 57.75 & 5.64 & 57.44 & -1.72 & - & & - & \\
\hline & ${ }^{2} J$ & $\Delta^{2} J$ & ${ }^{2} J$ & $\Delta^{2} J$ & ${ }^{2} J$ & $\Delta^{2} J$ & ${ }^{2} J$ & $\Delta^{2} J$ \\
\hline pcJ-1 & -1.08 & -6.49 & -0.13 & -4.57 & -0.25 & -5.13 & - & - \\
\hline co-r & -3.23 & -9.21 & 0.18 & -4.30 & 0.10 & -4.81 & 0.26 & -4.56 \\
\hline \multirow{2}{*}{ co-b } & -3.23 & -9.23 & 0.23 & -4.30 & - & - & - & - \\
\hline & ${ }^{3} J$ & $\Delta^{3} J$ & ${ }^{3} J$ & $\Delta^{3} J$ & ${ }^{3} J$ & $\Delta^{3} J$ & ${ }^{3} J$ & $\Delta^{3} J$ \\
\hline pcJ-1 & 10.72 & 5.38 & 5.87 & 2.47 & 6.36 & 3.52 & - & - \\
\hline co-r & 12.26 & 8.46 & 5.76 & 2.26 & 6.20 & 3.21 & 5.89 & 2.75 \\
\hline co-b & 12.41 & 8.53 & 5.80 & 2.28 & - & - & - & - \\
\hline
\end{tabular}

${ }^{a}$ All results for circumcircumcoronene were calculated with the locally dense co-r** basis set. See text for details. ${ }^{b}$ Conversion of the presently calculated absolute shielding constants to chemical shifts with respect to $\mathrm{CH}_{4}$ can be obtained by applying the formula $\delta=$ $188.33 \mathrm{ppm}-\sigma$.

converge, except $\mathrm{SD} / \mathrm{FC}$ in $\Delta^{3} J$, which exhibits some oscillation, with a $0.35 \mathrm{~Hz}$ difference between the results for the two largest systems.

The main limiting contribution to ${ }^{1,2,3} J$ arises again from the FC term, while the anisotropies are dominated by the SD/FC term, except in the case of $\Delta^{1} J$, for which there is a very near-cancellation of the PSO and the SD/FC terms. The ${ }^{2} J$ coupling constant is very close to zero in the larger molecules due to the fine balance of the small FC contribution with the other terms.

The magnitude of the HOMO-LUMO gap is seen to monotonically approach zero with the system size, as illustrated in the ESI $\dagger$. For the case of an infinite graphene sheet, a zero-gap semiconductor state would be attained. In a crude analysis, trends in the singlet and triplet excitation energies of relevance for the NMR properties can be approximated by the behavior of the HOMO-LUMO gap. From the second-order eqn (1), the fact that the results converge for these finite systems despite the absence of any plateau in the band gap (the denominator of the expression), implies that the matrix elements of the local hyperfine interactions (in the nominator) also decrease with the system size, cancelling the gap effect, and are accountable for the swift convergence of the investigated properties.

\section{Conclusions}

One-, two-, and three-bond ${ }^{13} \mathrm{C}-{ }^{13} \mathrm{C}$ spin-spin coupling and nuclear shielding constants, along with the anisotropies of the corresponding tensors with respect to the direction normal to the molecular plane, were calculated for planar hydrocarbon systems of increasing size. Ethene and benzene were used as 

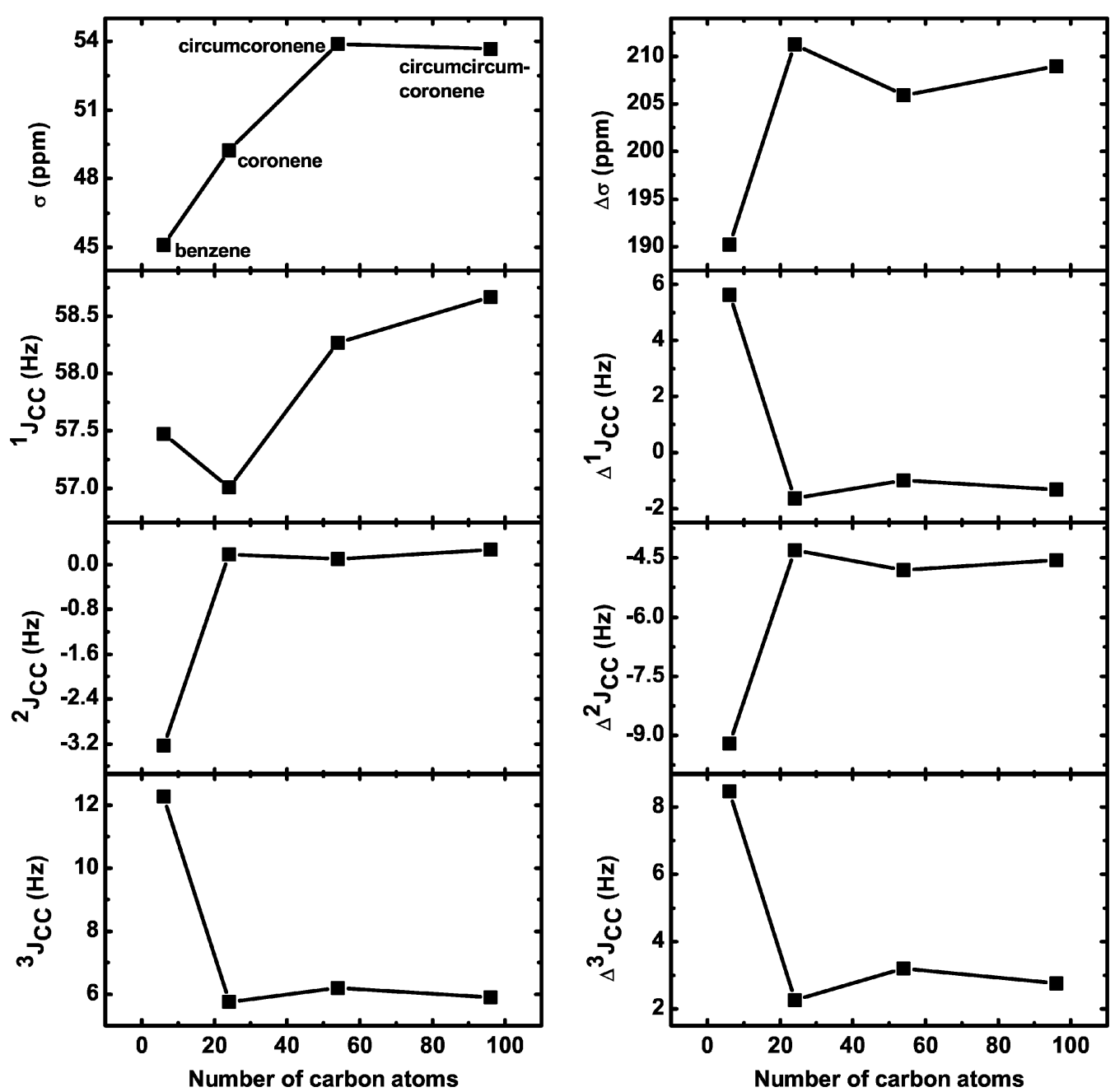

Fig. $6{ }^{13} \mathrm{C}$ nuclear shielding and ${ }^{13} \mathrm{C}-{ }^{13} \mathrm{C}$ spin-spin coupling constants, as well as the corresponding anisotropies for benzene, coronene, circumcoronene, and circumcircumcoronene with the PBE functional and the co-r basis set (co-r** for circumcircumcoronene).

test molecules, after which calculations were performed for benzene, coronene, circumcoronene, and circumcircumcoronene. It was found that the DFT functional PBE produces coupling data close to experimental results for ethene and benzene, as well as those obtained with the coupled-cluster singles and doubles method for ethene. The DFT shielding constants display the often-found systematic deshielding character. PBE was then used in calculations of the larger systems, namely coronene, circumcoronene, and circumcircumcoronene. Various basis set families were also tested, and the pcJ- $n$ and the novel completeness-optimized sets converge most rapidly for the spin-spin coupling. With a relatively small number of basis functions, the completeness-optimized basis sets generated in this work give results closer to converged values than the other basis set families, and allow close-to basisset-limit results to be obtained for the NMR parameters of the largest of the current systems. It is observed that with the PBE functional, the shielding constants and anisotropies, as well as the one-, two, and three-bond coupling constants and anisotropies within the innermost carbon hexagon approach limiting values as the number of carbon atoms is increased. These limiting values, which may be assigned to large planar hydrocarbons and finite carbon nanosheets, are (with error limits obtained by visual inspection) $\sigma=54 \pm 1 \mathrm{ppm}$, corresponding to the chemical shift $134 \mathrm{ppm}$ with $\mathrm{CH}_{4}$ as a reference, $\Delta \sigma=207 \pm 4 \mathrm{ppm}$, and for the couplings: ${ }^{1} \mathrm{~J}=$ $59.0 \pm 0.5 \mathrm{~Hz}, \Delta^{1} J=-1.5 \pm 0.5 \mathrm{~Hz},{ }^{2} J=0.2 \pm 0.4 \mathrm{~Hz}$, $\Delta^{2} J=-4.6 \pm 0.2 \mathrm{~Hz},{ }^{3} J=6 \pm 1 \mathrm{~Hz}$, and $\Delta^{3} J=3 \pm 1 \mathrm{~Hz}$. While these results strictly apply to the current choice of computational method only, the careful design of the basis set used, as well as the selection of the DFT functional should ensure the usefulness of the prediction for experimental analysis of large planar carbon systems, and eventually graphene.

\section{Acknowledgements}

We thank Dr Jyrki Hokkanen (CSC, Espoo, Finland) for significant help with the graphics. SI, PL, and JV are with the Finnish Center of Excellence in Computational Molecular Science (CMS). PL is an Academy Research Fellow of the Academy of Finland. JV is on leave from the University of Helsinki, Finland. Financial support was received from the National Graduate School of Computational Chemistry and 
Table 6 Calculated $J$ and $\Delta J$ for the ${ }^{13} \mathrm{C}-{ }^{13} \mathrm{C}$ spin-spin coupling tensor broken down into the different contributions (in $\mathrm{Hz}$ ) for benzene, coronene, circumcoronene, and circumcircumcoronene with the PBE functional and basis set co-r (co-r** for circumcircumcoronene). Couplings within the innermost carbon hexagon are considered. The HOMO-LUMO gaps of the systems are also provided

\begin{tabular}{|c|c|c|c|c|c|}
\hline Property & Term & $\mathrm{C}_{6} \mathrm{H}_{6}$ (gap $=0.191$ a.u. $)$ & $\mathrm{C}_{24} \mathrm{H}_{12}$ (gap $=0.105$ a.u. $)$ & $\mathrm{C}_{54} \mathrm{H}_{18}($ gap $=0.070$ a.u. $)$ & $\mathrm{C}_{96} \mathrm{H}_{24}$ (gap $=0.050$ a.u. $)$ \\
\hline \multirow[t]{5}{*}{$\overline{1} J$} & Total & 57.47 & 57.01 & 58.27 & 58.67 \\
\hline & DSO & 0.22 & 0.40 & 0.43 & 0.44 \\
\hline & PSO & -6.96 & -5.60 & -5.75 & -5.67 \\
\hline & SD & 1.32 & 0.74 & 0.79 & 0.77 \\
\hline & $\mathrm{FC}$ & 62.89 & 61.47 & 62.79 & 63.13 \\
\hline \multirow[t]{5}{*}{$\Delta^{1} J$} & Total & 5.63 & -1.64 & -1.00 & -1.32 \\
\hline & DSO & -2.08 & -1.79 & -1.80 & -1.78 \\
\hline & PSO & 10.54 & 7.93 & 8.05 & 7.88 \\
\hline & SD & 1.42 & 0.10 & 0.23 & 0.19 \\
\hline & $\mathrm{SD} / \mathrm{FC}$ & -4.25 & -7.88 & -7.49 & -7.61 \\
\hline \multirow[t]{5}{*}{${ }^{2} J$} & Total & -3.23 & 0.18 & 0.10 & 0.26 \\
\hline & DSO & -0.02 & 0.11 & 0.13 & 0.14 \\
\hline & PSO & 0.07 & -0.08 & -0.11 & -0.10 \\
\hline & SD & -0.79 & -0.21 & -0.28 & -0.24 \\
\hline & $\mathrm{FC}$ & -2.49 & 0.36 & 0.35 & 0.47 \\
\hline \multirow[t]{5}{*}{$\Delta^{2} J$} & Total & -9.21 & -4.30 & -4.81 & -4.56 \\
\hline & DSO & -0.48 & -0.36 & -0.35 & -0.34 \\
\hline & PSO & 0.12 & 0.09 & 0.08 & 0.06 \\
\hline & $\mathrm{SD}$ & -1.07 & -0.23 & -0.32 & -0.27 \\
\hline & $\mathrm{SD} / \mathrm{FC}$ & -7.78 & -3.81 & -4.22 & -4.01 \\
\hline \multirow[t]{5}{*}{${ }^{3} J$} & Total & 12.26 & 5.76 & 6.20 & 5.89 \\
\hline & DSO & -0.01 & 0.10 & 0.13 & 0.13 \\
\hline & PSO & 0.53 & -0.01 & 0.00 & -0.02 \\
\hline & SD & 1.97 & 0.54 & 0.75 & 0.63 \\
\hline & $\mathrm{FC}$ & 9.77 & 5.14 & 5.33 & 5.15 \\
\hline \multirow{5}{*}{$\Delta^{3} J$} & Total & 8.46 & 2.26 & 3.21 & 2.75 \\
\hline & DSO & -0.31 & -0.21 & -0.20 & -0.19 \\
\hline & PSO & -0.86 & -0.33 & -0.41 & -0.36 \\
\hline & SD & 2.86 & 0.83 & 1.13 & 0.97 \\
\hline & $\mathrm{SD} / \mathrm{FC}$ & 6.77 & 1.98 & 2.69 & 2.34 \\
\hline
\end{tabular}

Molecular Spectroscopy as well as the Alfred Kordelin Fund (SI). Computational resources were partially provided by the CSC-IT Center for Science Ltd., Espoo, Finland.

\section{References}

1 A. Abragam, The Principles of Nuclear Magnetism, Oxford University Press, Oxford, 1961.

2 C. P. Slichter, Principles of Magnetic Resonance, Springer, Berlin, 2nd edn, 1990

3 K. S. Novoselov, A. K. Geim, S. V. Morozov, D. Jiang, Y. Zhang, S. V. Dubonos, I. V. Grigorieva and A. A. Firsov, Science, 2004 306, 666 .

4 C. Goze-Bac, S. Latil, P. Lauginie, V. Jourdain, J. Conard, L. Duclaux, A. Rubio and P. Bernier, Carbon, 2002, 40, 1825.

5 E. Kan, Z. Li and J. Yang, Nano, 2008, 3, 433

6 A. H. Castro Neto, F. Guinea, N. M. R. Peres, K. S. Novoselov and A. K. Geim, Rev. Mod. Phys., 2009, 81, 109.

7 P. R. Wallace, Phys. Rev., 1947, 71, 622.

8 B. Dóra and F. Simon, Phys. Rev. Lett., 2009, 102, 197602.

9 J. Conard, H. Estrade, P. Lauginie, H. Fuzellier, G. Furdin and R. Vasser, Physica B+C, 1980, 99, 521.

10 Y. Hiroyama and K. Kume, Solid State Commun., 1988, 65, 617.

11 F. Mauri, B. G. Pfrommer and S. G. Louie, Phys. Rev. Lett., 1996, 77, 5300 .

12 D. Sebastiani and M. Parrinello, J. Phys. Chem. A, 2001, 105, 1951.

13 S. A. Joyce, J. R. Yates, C. J. Pickard and F. Mauri, J. Chem. Phys., 2007, 127, 204107.

14 T. Helgaker, M. Jaszuński and K. Ruud, Chem. Rev., 1999, 99, 293.

15 J. Vaara, Phys. Chem. Chem. Phys., 2007, 9, 5399.

16 J. Kaski, P. Lantto, J. Vaara and J. Jokisaari, J. Am. Chem. Soc., 1998, 120, 3993.

17 J. Kaski, J. Vaara and J. Jokisaari, J. Am. Chem. Soc., 1996, 118, 8879 .
18 A. M. Orendt, J. C. Facelli, S. Bai, A. Rai, M. Gossett, L. T. Scott, J. Boerio-Goates, R. J. Pugmire and D. M. Grant, J. Phys. Chem. A, 2000, 104, 149.

19 M. Jaszuński, K. Ruud and T. Helgaker, Mol. Phys., 2003, 101, 1997.

20 E. Zurek and J. Autschbach, J. Am. Chem. Soc., 2004, 126, 13079.

21 P. Manninen and J. Vaara, J. Comput. Chem., 2006, 27, 434.

22 F. Jensen, J. Chem. Theory Comput., 2006, 2, 1360.

23 N. F. Ramsey, Phys. Rev., 1953, 91, 303.

24 N. F. Ramsey, Phys. Rev., 1950, 78, 695.

25 K. Wolinski, J. F. Hinton and P. J. Pulay, J. Am. Chem. Soc., 1990, 112, 8251 .

26 T. Helgaker and P. Jørgensen, J. Chem. Phys., 1991, 95, 2595.

27 J. Oddershede, J. Geertsen and G. E. Scuseria, J. Phys. Chem., 1988, 92, 3056 .

28 T. Helgaker, M. Jaszuński, K. Ruud and A. Górska, Theor. Chem. Acc., 1998, 99, 175.

29 T. Enevoldsen, J. Oddershede and S. P. A. Sauer, Theor. Chem. Acc., 1998, 100, 275.

30 J. Vaara, J. Jokisaari, R. E. Wasylishen and D. L. Bryce, Prog. Nucl. Magn. Reson. Spectrosc., 2002, 41, 233.

31 T. Helgaker, M. Jaszuński and M. Pecul, Prog. Nucl. Magn. Reson. Spectrosc., 2008, 53, 249

32 F. Jensen, J. Chem. Phys., 2001, 115, 9113.

33 D. P. Chong, Can. J. Chem., 1995, 73, 79.

34 S. Ikäläinen, P. Lantto, P. Manninen and J. Vaara, J. Chem. Phys., 2008, 129, 124102.

35 P. Manninen, Kruununhaka ver. 1.2. http://www.chem.helsinki.fi/ $\sim$ manninen/kruununhaka.

36 Dalton, Release 2.0, (2005). http://www.kjemi.uio.no/software/ dalton/dalton.html.

37 J. F. Stanton, J. Gauss, J. D. Watts, P. G. Szalay and R. J. Bartlett, with contributions from A. A. Auer, D. E. Bernholdt, O. Christiansen, M. E. Harding, M. Heckert, O. Heun, C. Huber, D. Jonsson, J. Jusélius, W. J. Lauderdale, T. Metzroth, C. Michauk, D. P. O'Neill, D. R. Price, K. Ruud, F. Schiffmann, M. E. Varner, J. Vázquez and the integral packages 
MOLECULE (J. Almlöf and P. R. Taylor), PROPS (P. R. Taylor) and ABACUS (T. Helgaker, H. J. Aa. Jensen, P. Jørgensen and J. Olsen). For the current version, see http://www.aces2.de.

38 A. D. Becke, Phys. Rev. A: At., Mol., Opt. Phys., 1988, 38, 3098.

39 C. Lee, W. Yang and R. G. Parr, Phys. Rev. B: Condens. Matter, 1988, 37, 785.

40 D. E. Woon and T. H. Dunning Jr, J. Chem. Phys., 1995, 103, 4572.

41 D. B. Chesnut and K. D. Moore, J. Comput. Chem., 1989, 10, 648

42 D. B. Chesnut, B. E. Rusiloski, K. D. Moore and D. A. Egolf Jr, J. Comput. Chem., 1993, 14, 1364.

43 Electronic Structure Calculations on Workstation Computers: The Program System TURBOMOLE; R. Ahlrichs, M. Bär, M. Häser, H. Horn and C. Kölmel, Chem. Phys. Lett., 1989, 162, 165.

44 A. D. Becke, J. Chem. Phys., 1993, 98, 5648.

45 P. Stephens, F. Devlin, C. Chabalowski and M. J. Frisch, J. Phys. Chem., 1994, 98, 11623.

46 A. D. Becke, J. Chem. Phys., 1993, 98, 1372.

47 J. P. Perdew, K. Burke and M. Ernzerhof, Phys. Rev. Lett., 1996, 77, 3865; erratum, 1997, 78, 1396.

48 V. G. Malkin, O. L. Malkina and D. R. Salahub, Chem. Phys. Lett., 1994, 221, 91.

49 P. Lantto, J. Vaara and T. Helgaker, J. Chem. Phys., 2002, 117, 5998.

50 T. Helgaker, O. B. Lutnæs and M. Jaszuński, J. Chem. Theory Comput., 2007, 3, 86.
51 J. M. L. Martin and P. R. Taylor, Chem. Phys. Lett., 1996, 248, 336.

52 J. Gauss and J. F. Stanton, J. Phys. Chem. A, 2000, 104, 2865.

53 J. P. Perdew, Phys. Rev. B: Condens. Matter, 1986, 33, 8822.

54 F. Weigend and R. Ahlrichs, Phys. Chem. Chem. Phys., 2005, 7, 3297.

55 T. H. Dunning Jr, J. Chem. Phys., 1989, 90, 1007.

56 R. A. Kendall, T. H. Dunning Jr and R. J. Harrison, J. Chem. Phys., 1992, 96, 6796.

57 K. Jackowski, E. Maciąga and M. Wilcek, J. Mol. Struct., 2005, 101, 744.

58 A. Pines, M. G. Gibby and J. S. Waugh, Chem. Phys. Lett., 1972, $15,373$.

59 J. R. Cheeseman, G. W. Trucks, T. A. Keith and M. J. Frisch, J. Chem. Phys., 1996, 104, 5497.

60 G. Rauhut, S. Pulyear, K. Wolinski and P. Pulay, J. Phys. Chem., 1996, 100, 6310.

61 A. K. Jameson and C. J. Jameson, Chem. Phys. Lett., 1987, 134, 461.

62 R. D. Johnson, G. Meijer and D. S. Bethune, J. Am. Chem. Soc., 1990, 112, 8983

63 R. Taylor, J. P. Hare, A. K. Abdul-Sada and H. W. Kroto, Chem. Commun., 1990, 1423.

64 C. S. Yannoni, R. D. Johnson, G. Meijer, D. S. Bethune and J. R. Salem, J. Phys. Chem., 1991, 95, 9. 